\title{
PAPR Reduction in OFDM Systems using Non-Linear Companding Transform
}

\author{
Deepender Gill ${ }^{1}$, Garima Saini ${ }^{2}$ \\ ${ }^{I}$ (P.G. Scholar, Department of ECE/ NITTTR Chandigarh, India) \\ ${ }^{2}$ (Assistant Professor, Department of ECE/ NITTTR Chandigarh, India)
}

\begin{abstract}
In this paper a non-linear companding transform is proposed which can effectively reduce the PAPR value in OFDM systems. High value of PAPR has undesirable effects on the performance of wireless communication systems. Various companding schemes are used to reduce the problem of PAPR in orthogonal frequency division multiplexing schemes. High PAPR drives the power amplifiers used at the transmitter's side into non-linear region and leads to high power dissipation in hand held devices. The proposed NCT has the capability to transform power and amplitude of the original OFDM signal into a uniform distribution which leads to the reduction of PAPR.

Keywords :Complementary Cumulative Distribution Function $(C C D F)$, Inverse Fast Fourier Transform(IFFT),Non-linear Companding Transform(NCT),Orthogonal Frequency division Multiplexing(OFDM),Peak-to-average power ratio(PAPR), ,Quadrature Amplitude Modulation $(Q A M)$.
\end{abstract}

\section{Introduction}

OFDM is a multicarrier modulation technique that divides the available spectrum among various low data rate subcarriers. Orthogonality is a must for OFDM otherwise it will lead to spectral overlapping. The subcarriers are so spaced as to satisfy the orthogonality condition [1]. A guard band is also provided in OFDM by using cyclic extension to avoid ICI. Frequency selective fading can also be decreased in OFDM by increasing the number of sub carriers. OFDM can provide an adaptive and scalable technology for wireless communication.

Due to the advent of $3 \mathrm{G}$ and $4 \mathrm{G}$ services the demand for high data rates in wireless communication is constantly increasing. One of the main focuses of the $4 \mathrm{G}$ systems is to significantly improve the spectral efficiency which makes OFDM as a potential candidate for the physical layer of $4 \mathrm{G}$ systems. OFDM makes efficient usage of the bandwidth [2]. Apart from spectral efficiency OFDM has many other advantages like being robust to multipath fading, interchannel interference and co-channel interference. Due to these inherent advantages OFDM is used in many wireless standards like IEEE 802.11a and IEEE802.16a [3]. OFDM is a multi carrier transmission scheme which divides the allocated spectrum into many parallel orthogonal subchannels. High rate data stream is split into many lower rate data streams which are transmitted at the same time over these equally spaced frequency bands. A subcarrier carrying a portion of the user information is transmitted in each band. The subcarriers are overlapping but they are recoverable since they are orthogonal [4].

\section{Ofdm System Model}

In OFDM each sub-carrier to be produced is assigned some data to transmit. The required amplitude and phase is calculated based on the modulation scheme. The digital data stream in serial form is converted to parallel form using a serial to parallel convertor. Inverse Fourier Transform (IFT) is used to convert back the required spectrum to its time domain signal an as shown Fig. 1. In most applications, an Inverse Fast Fourier Transform (IFFT) is used [5]. The IFFT block performs the time domain transformation very efficiently and provides a simple way of ensuring the carrier signals produced are orthogonal to each other. The output of the IFFT is provided as an input to the compander. The companded data is converted to analog form and transmitted after amplification through a high power amplifier (HPA).At the receiver side the data is converted back to digital form and allied to a decompander [6]. 


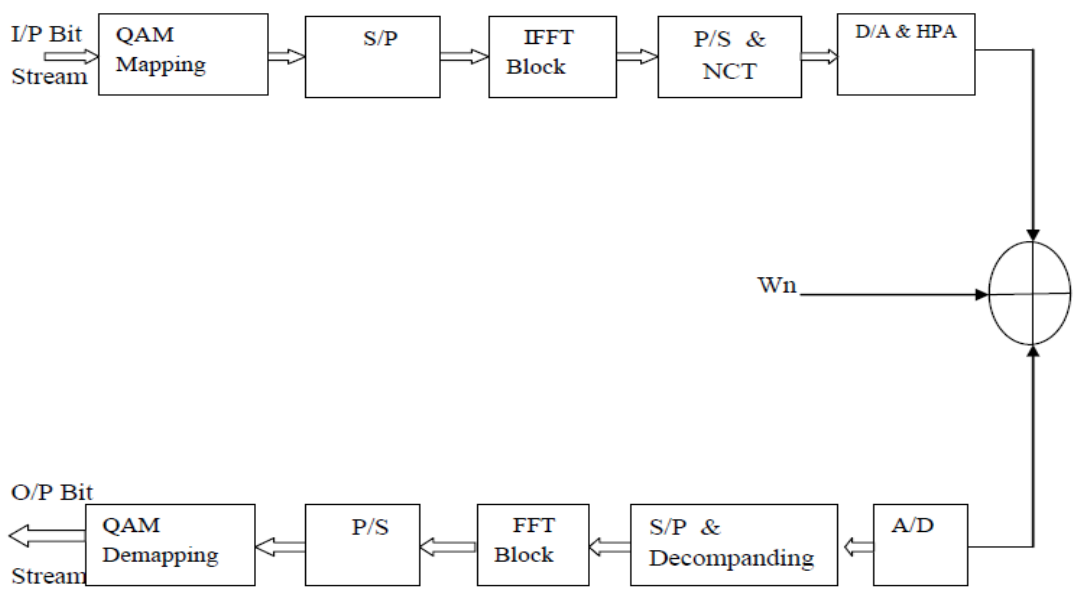

Fig. 1 OFDM System Model

This paper deals with formulating the companding transform. This NCT is to be used in the companding block at the transmitter side. The transmitter side of the OFDM system has been simulated for observing the PAPR introduced during the generation of OFDM signal.

\section{Peak-to-Average-Power Ratio}

The reason for high PAPR in OFDM systems is that in time domain, a multicarrier signal is the sum of many narrow-band signals. At some time instances, this sum is large and at other times it is small which means that the peak value of the signal is substantially larger than the average value[7]. The peak to average ratio for a signal $\mathrm{x}(\mathrm{t})$ may be defined as

$\mathbf{P A P R}=\max \left[\mathbf{x}(\mathbf{t}) \mathbf{x}^{*}(\mathbf{t})\right] / \mathrm{E}\left[\mathbf{x}(\mathbf{t}) \mathbf{x}^{*}(\mathbf{t})\right]$

In decibels PAPR may be expressed as

PAPR $_{\mathrm{dB}}=10 \log 10$ (PAPR)

Let $\mathrm{N}$ be used to denote the number of sub-carriers used for the transmission of parallel information in the OFDM system. Let the $\mathrm{K}^{\text {th }}$ modulated symbol in a block of $\mathrm{N}$ information symbols be denoted by $\mathrm{S}_{\mathrm{K}}$ where $0<\mathrm{K}<\mathrm{N}-1$. IFFT of this complex modulated symbol is performed to generate the OFDM signal. Mathematically it can be expressed as[8].

$S_{\mathbf{n}}=1 / \sqrt{ } \mathbf{N} \sum_{k=0}^{N-1} S K \exp \left(\mathbf{j} 2 \prod \mathrm{kn} / \mathrm{N}\right)$

According to the central limit theorem for large values of $N$ the distribution of $S_{n}$ is Gaussian whose probability distribution function (pdf) may be given as

$\mathbf{f}_{\mathrm{Sn}}(\mathbf{s})=1 / \sqrt{ } \mathbf{2} \prod \sigma \exp \left(\mathrm{s}^{2} / 2 \sigma\right)$

where $\sigma^{2}$ is the variance

The symbols used as input are assumed to be statistically independent from each other and are also assumed to be identically distributed. When the number of subcarriers is large then real and imaginary parts of $S_{n}$, are identically distributed random variables which have zero mean and common variance denoted as $\sigma^{2}$ according to the central limit theorem. Therefore amplitude of the signal $S_{n}$ may be givan as

$\left|\mathbf{S}_{\mathbf{n}}\right|=\sqrt{ } \operatorname{Re}^{2}\left\{\mathbf{S}_{\mathbf{n}}\right\}+\operatorname{Im}^{2}\left\{\mathbf{S}_{\mathbf{n}}\right\}$

The cumulative distribution function C.D.F for the signal $S_{n}$ is given as

$\mathbf{F}_{|\mathrm{Sn}|}(\mathbf{x})=\operatorname{Prob}\left\{\left|\mathbf{S}_{\mathrm{n}}\right|<=\mathbf{x}\right\}$

$$
\begin{aligned}
& =\int_{0}^{x}\left(2 \cdot y / \sigma^{2}\right) \exp \left(-y^{2} / \sigma^{2}\right) d y \\
& =1-\exp \left(-x^{2} / \sigma^{2}\right), \quad x=>0
\end{aligned}
$$

The amplitude given by the expression is rayleigh distributed. The power of the signal $\mathrm{S}_{\mathrm{n}}$ may be calculated as $\left|S_{n}\right|^{2}=1 / N \sum_{m=0}^{N-1} \sum_{k=0}^{N-1} \operatorname{SmSk} \exp (\mathbf{j} .2 \pi(m-k) \cdot \mathbf{n} / \mathrm{N})$

The PAPR for the OFDM signal $S_{n}$ is the ratio of the maximum power of the signal to its average power. It is expressed in dBs. In one symbol period the PAPR for the OFDM signal may be defined as

PAPR $=\operatorname{10log}_{10}\left[\operatorname{Max}\left\{\left|\mathbf{S}_{\mathbf{n}}\right|^{2}\right\}\right] /\left[\mathbf{E}\left\{\left|\mathbf{S}_{\mathbf{n}}\right|^{2}\right\}\right] \mathrm{dB}$

Due to inherent disadvantages of high PAPR it needs to be reduced before the OFDM signal is to be transmitted through the channel. Many techniques have been proposed in the literature to reduce high value of PAPR in OFDM signals. Block Coding, Selective Mapping and Partial Transmit Sequence are some of the techniques which may be used for the reduction of high PAPR in OFDM. The drawback of these techniques is that they put some restrictions on various parameters used in the OFDM system such as frame format, constellation size etc [9].The signal distortion technique for the reduction of PAPR suitable for high data rates is 
companding. Companding is a combination of compression and expansion [10]. The reverse process of companding is known as decompanding. For PAPR reduction in OFDM system companding is done at the transmitter side and the decomapnding is done at the receiver side. Companding is basically the process of making quantization levels unequal. In companding the quantization levels are kept very close for the small amplitude signals and larger spacing between quantization levels is used for high amplitude signals [11].

\section{FORMULATION Of COMPANDING-TRANSFORM}

In this paper non-linear companding transform technique is used for reducing the PAPR of OFDM signal. The companding function transforms the statistics of the amplitude of the signals into a uniform distribution. The companded signal has almost constant average power level which relieves the strict linearity requirements on the HPA (High Power Amplifiers) used in the process.

The companding function can transform the original signal $\mathrm{x}_{\mathrm{n}}$ into a form which leads to the reduction of PAPR. In the interval $[0, \mathrm{~K}]$ where $\mathrm{k}>0$ the target PDF of the transformed signal may be defined as

$$
\mathbf{f}_{|y|}(x)= \begin{cases}\mathbf{p T ~ x}^{\mathbf{q}} & \mathbf{0}<=x<=\mathbf{m T} \\ \mathbf{p} / \mathbf{T}(\mathbf{m T})^{\mathbf{q}} & \mathbf{m T}<=x<=T\end{cases}
$$

The cumulatize distribution function for the above PDF may be calculated as

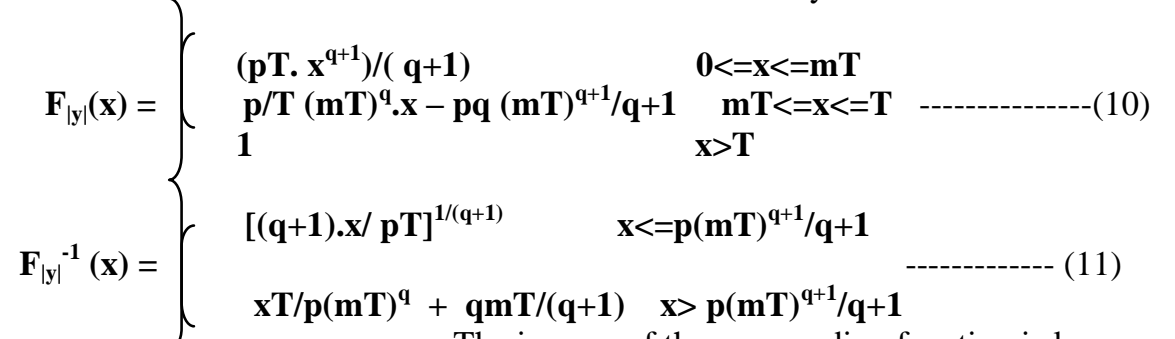

inverse of the companding function is known as don and is used at the receiver's side to bring the signal back to its original form. The companding function $\mathrm{h}(\mathrm{x})$ has to be monotoniqally increasing function in order for it to be exactly invertible [12]. The proposed companding functions fulfilsthis condition and hence its inverse can be used for decompanding at the receivers side. The compnding function $\mathrm{h}(\mathrm{x})$ can be calculated as

$$
\mathbf{h}(\mathbf{x})=\mathbf{F}_{|\mathbf{y}|}^{-1}\left[\mathbf{F}_{\mid \mathbf{x}}(\mathbf{x})\right] \cdot \operatorname{sgn}(\mathbf{x})---
$$

Where $\mathrm{F}_{|\mathrm{x}|}(\mathrm{x})$ is the cumulative distribution function for the input signal $\mathrm{x}$ and may be given as

$\mathbf{F}_{|x|}(\mathbf{x})=1-\exp \left(-\mathbf{x}^{2} / \sigma^{2}\right)$

By using these expressions the companding function may be derived as

$$
\mathbf{h}(\mathbf{x})=\left\{\begin{array}{l}
{\left[(\mathbf{q}+1) \mathbf{x} / \mathbf{p T} \cdot\left(\mathbf{F}_{|\mathbf{x}|}(\mathbf{x})\right)\right]^{1 /(\mathbf{q}+1)} \cdot \operatorname{sgn}(\mathbf{x})} \\
{\left[\mathbf{T} \cdot \mathbf{F}_{|\mathbf{x}|}(\mathbf{x}) / \mathbf{p}(\mathbf{m T})^{\mathrm{m}}+\mathbf{q c T} /(\mathbf{q}+\mathbf{1}) \cdot \operatorname{sgn}(\mathbf{x})\right.}
\end{array}\right.
$$

The proposed companding function has the ability to compress large amplitude signals and at the same time expand the small amplitude signals which is the primary requirement for PAPR reduction. The proposed function provides the flexibility and some adjustable parameters which can be changed to obtain different profiles for the companding transform.

\section{Simulation Results}

OFDM transmitter block has been simulated using matlab. Figure (2) shows the OFDM signal using 16QAM mapping in time and frequency domain before the application of NCT. High value of PAPR occurs due to difference between the peak value of the signal and the average value of the signal. The purpose of applying the NCT is to reduce the ratio of peak value to the average value. Simulation has been done using $6.4 \times 10^{5}$ data points. 

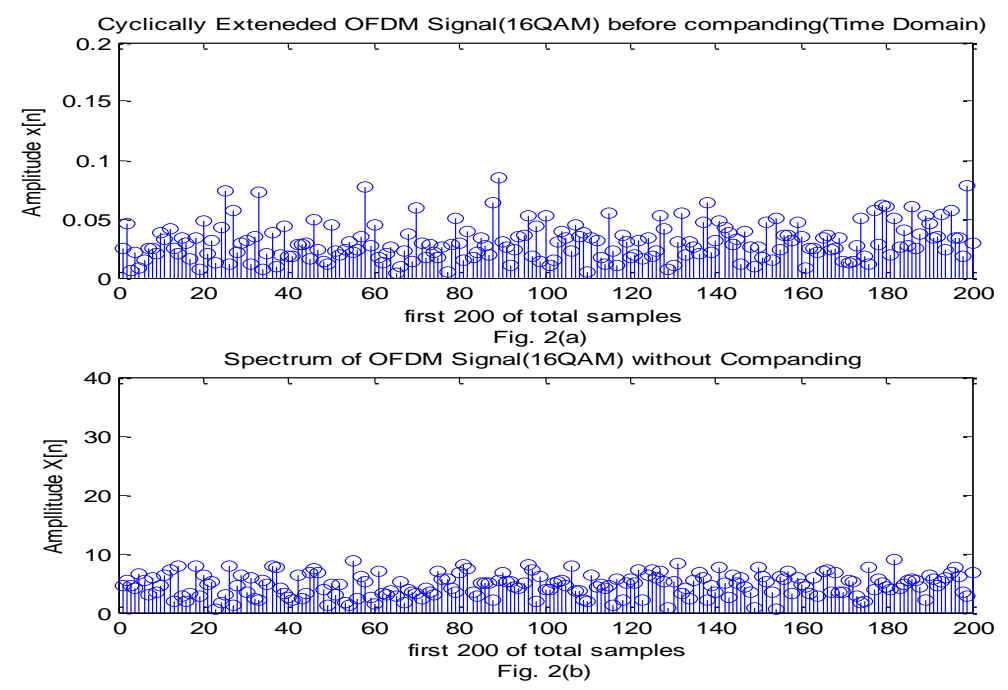

Fig.2 OFDM Signal (16 QAM) before companding

Fig. 3 shows a plot of CCDF vs PAPR for the OFDM signal before the application of NCT .The peak value of PAPR goes to 11.8.The original OFDM signal has a high value of PAPR which is to be brought down by the application of NCT.

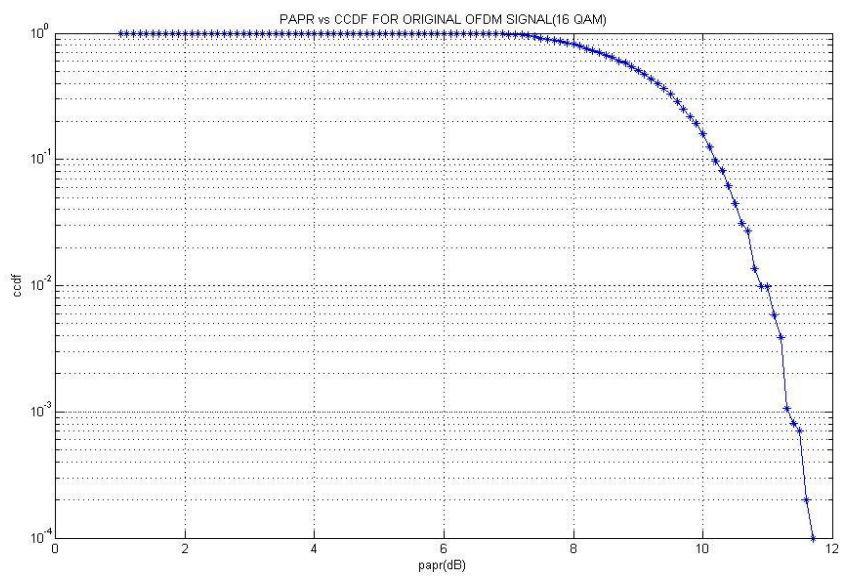

Fig.3 PAPR vs CCDF for original OFDM signal

Fig.4. shows the companding function for different values of $\mathrm{q}$ and $\mathrm{m}$. Values used are

(i) $\quad \mathrm{q}=0.5, \mathrm{~m}=0.20$

(ii) $\quad \mathrm{q}=0.5, \mathrm{~m}=0.5$

(iii) $\mathrm{q}=0.5, \mathrm{~m}=0.25$

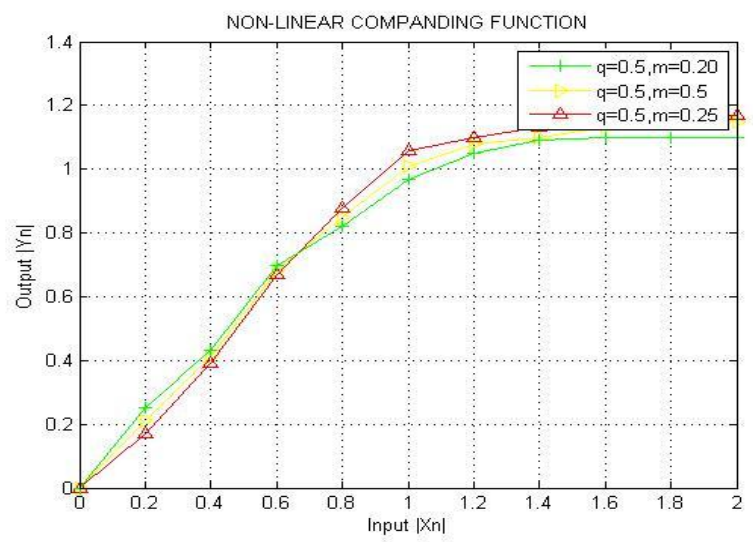

Fig.4. Transfer curve of NCT with different parameters 


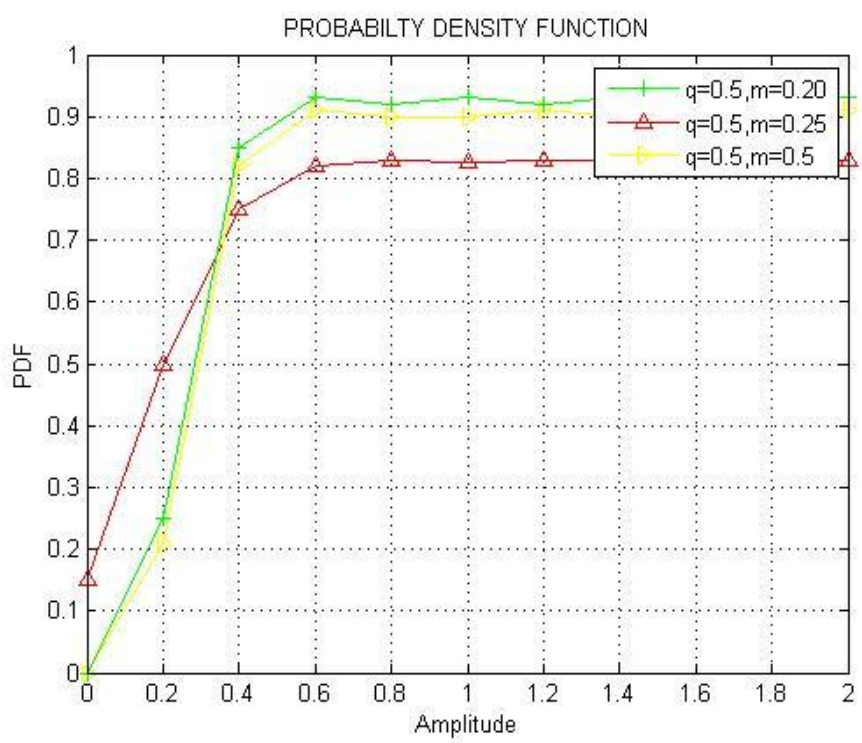

Fig.5 PDF Profile

Fig.5. shows the PDF profile of the companded signal for different values of $\mathrm{q}$ and $\mathrm{m}$

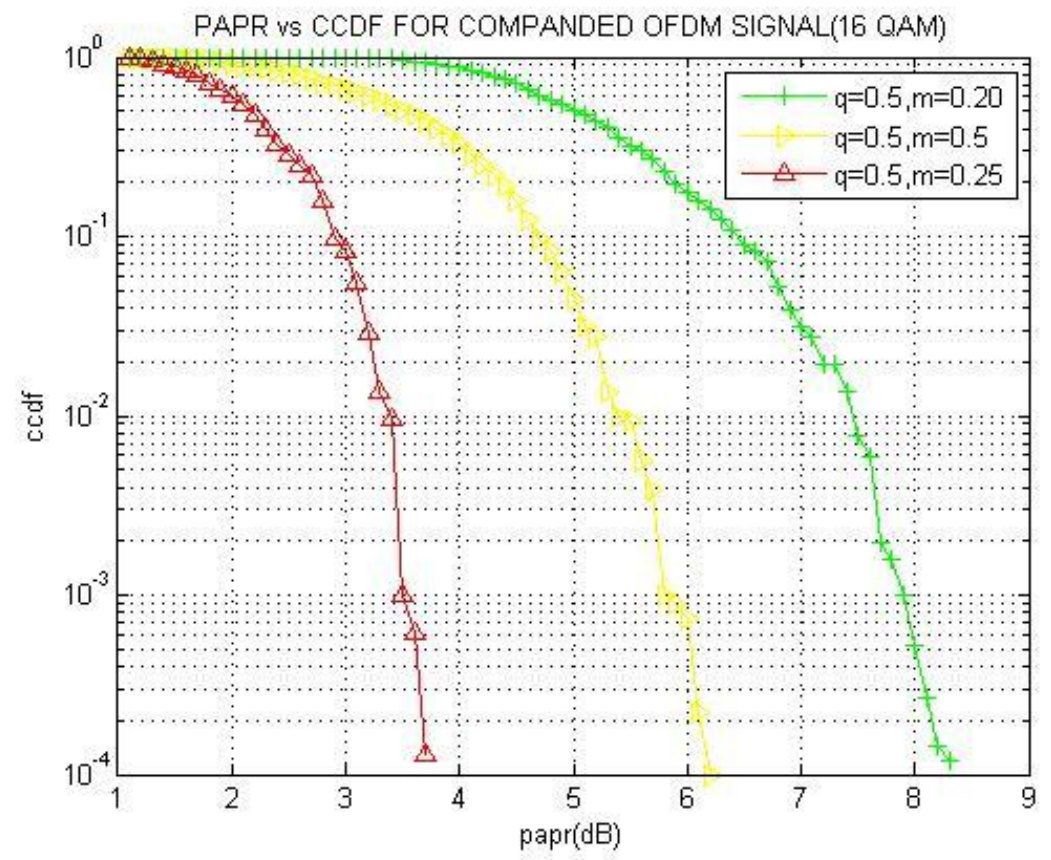

Fig.6 PAPR vs CCDF curves for the transformed signal(16 QAM)

Fig. 6. shows PAPR vs CCDF plot for the OFDM signal 16 QAM after the application of NCT. Figure shows the PAPR vs CCDF plots for the companded OFDM Signal. The graph has been plotted corresponding to the three sets of values.

\begin{tabular}{|c|c|c|c|}
\hline S.No. & q & m & PAPR Value(in dB) \\
\hline 1 & 0.5 & 0.2 & 8.25 \\
\hline 2 & 0.5 & 0.5 & 6.2 \\
\hline 3 & 0.5 & 0.25 & 3.75 \\
\hline
\end{tabular}

Table 1

The application of NCT leads to significant improvement in the PAPR value of the OFDM signal as compared to the original OFDM signal. Table 1 shows that the application of NCT can bring down the PAPR value to 3.75 $\mathrm{dB}$ which was $11.8 \mathrm{~dB}$ in case of original OFDM signal using 16 QAM mapping. 

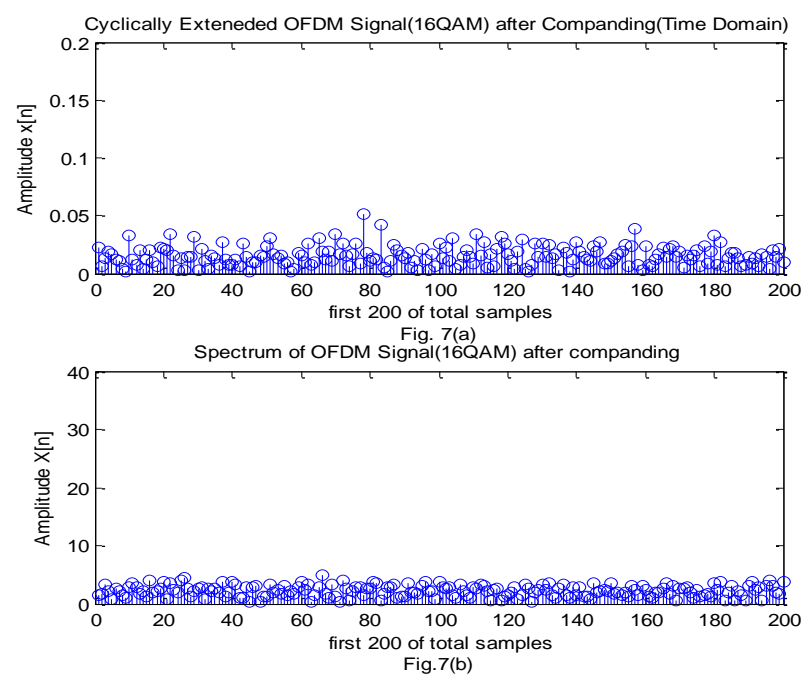

Fig.7 OFDM Signal (16 QAM) after companding

Fig. 7. shows the time and frequency domain representation of the companded OFDM signal. The application of NCT has a smoothing effect on the OFDM signal. This is achieved by the NCT by enlarging the small amplitude signals and at the same time compressing the large amplitude signals.
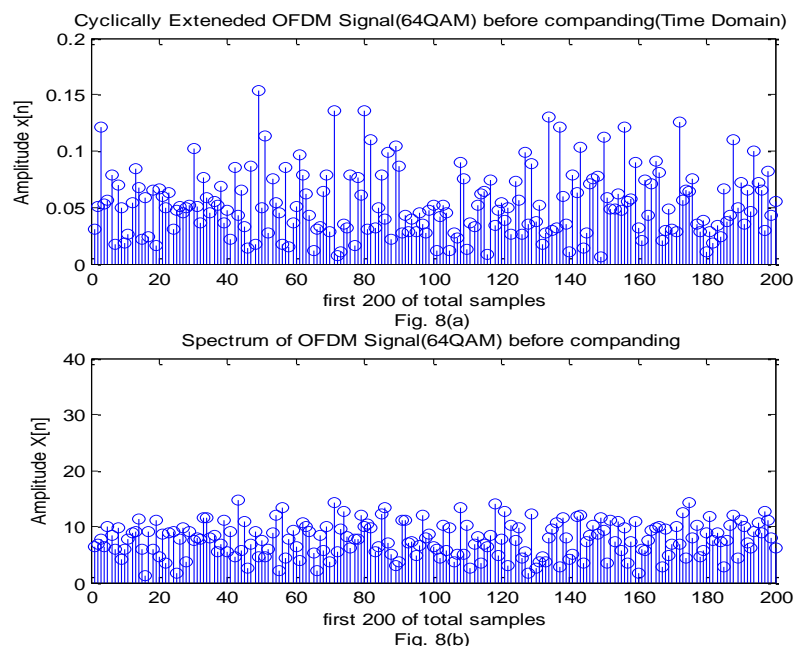

Fig.8 OFDM Signal(64 QAM) before companding

Fig. 8.shows the OFDM signal using 64 QAM mapping in time and frequency domain before the application of NCT.

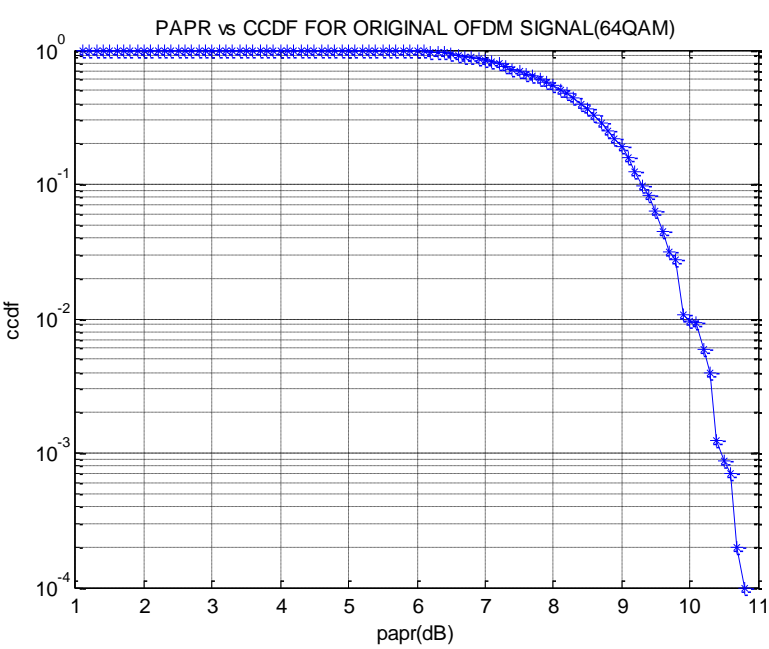

Fig.9 PAPR vs CCDF for original OFDM signal(64 QAM) 
Fig. 9 shows a plot of CCDF vs PAPR for the OFDM signal using 64 QAM mapping before the application of NCT .The peak value of PAPR goes to 10.85.The original OFDM signal has a high value of PAPR which is to be brought down by the application of NCT. The companding function which was earlier used to transform an OFDM signal using 16 QAM mapping is being used here to transform an OFDM signal using 64 QAM mapping. Different constellation sizes have used to check the flexibility of NCT.

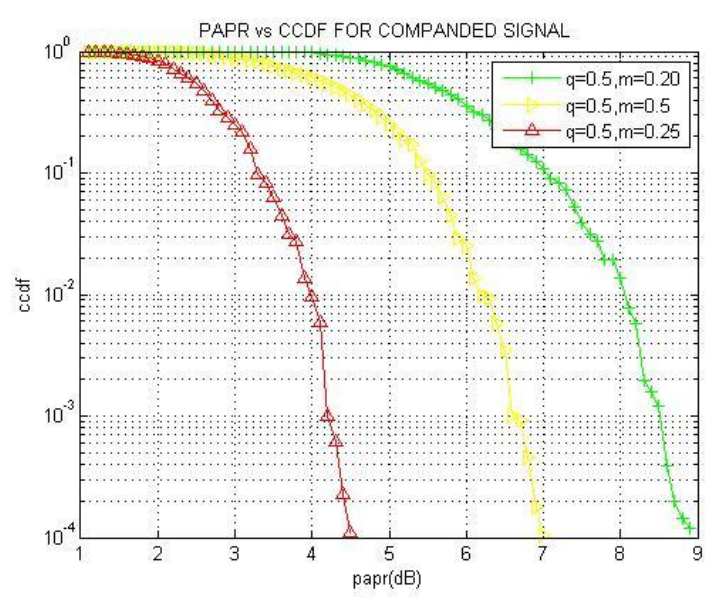

Fig.10 PAPR vs CCDF curves for the transformed signal(64 QAM)

Fig. 10 shows PAPR vs CCDF plot for the OFDM signal 64 QAM after the application of NCT. Figure shows the PAPR vs CCDF plots for the companded OFDM Signal. The graph has been plotted corresponding to the three sets of values.

\begin{tabular}{|c|c|c|c|}
\hline S.No. & q & m & PAPR Value(in dB) \\
\hline 1 & 0.5 & 0.2 & 9.0 \\
\hline 2 & 0.5 & 0.5 & 6.9 \\
\hline 3 & 0.5 & 0.25 & 4.4 \\
\hline
\end{tabular}

Table 2

The application of NCT leads to significant improvement in the PAPR value of the OFDM signal as compared to the original OFDM signal. Table 2 shows The application of NCT can bring down the PAPR value to $4.4 \mathrm{~dB}$ which was $10.85 \mathrm{~dB}$ in case of original OFDM signal using 64 QAM mapping.
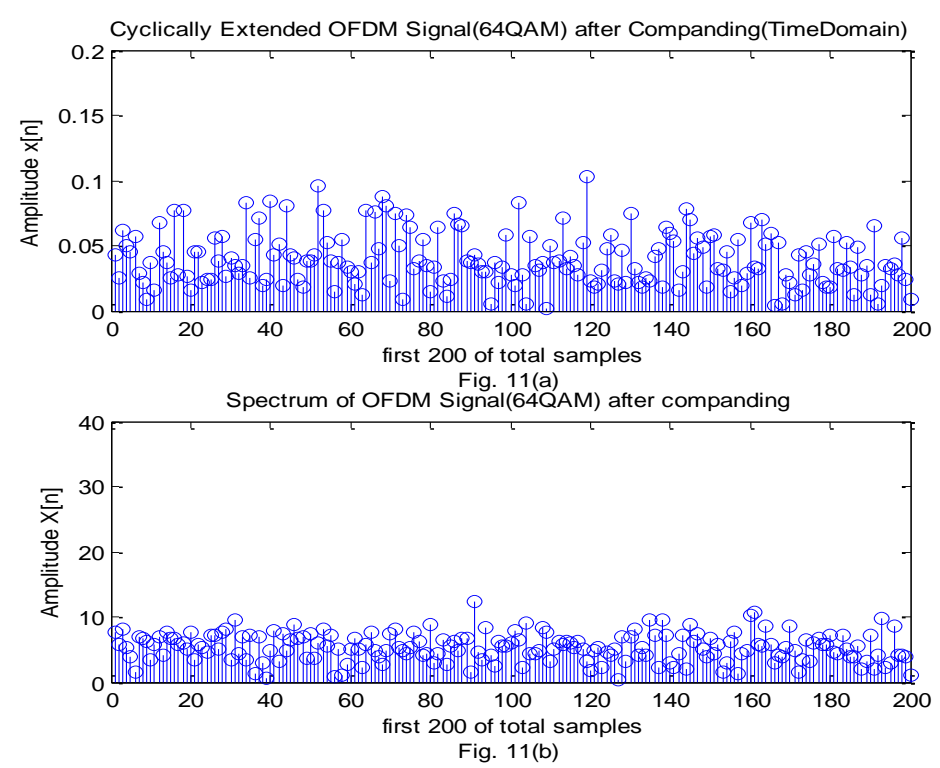

Fig.11 OFDM Signal(64 QAM) after companding

Fig.11. shows the time and frequency domain representation of the companded OFDM signal. The application of NCT has a smoothing effect on the OFDM signal. This is achieved by the NCT by enlarging the small amplitude signals and at the same time compressing the large amplitude signals. 


\section{Comparison}

Table 3 gives a comparison of various PAPR reduction techniques.. Various PAPR reduction techniques and their reduced PAPR value results have been tabulated. In terms of PAPR reduction the proposed NCT provides improvement over other techniques

\begin{tabular}{|l|l|l|l|}
\hline S.No & $\begin{array}{l}\text { Name of PAPR Reduction } \\
\text { Technique }\end{array}$ & $\begin{array}{l}\text { Improved PAPR after } \\
\text { application of PAPR Reducion } \\
\text { Technique (in dB) }\end{array}$ & Source \\
\hline 1. & Tone Reservation & 10.5 & {$[13]$} \\
\hline 2. & PTS & 8.5 & {$[18]$} \\
\hline 3. & Modified Exponential & 6.1 & {$[17]$} \\
\hline 4. & $\mu$-law & 5.58 & {$[9]$} \\
\hline 5. & Trapeziodal companding & 5.24 & {$[15]$} \\
\hline 6. & Proposed NCT & 3.75 & \\
\hline
\end{tabular}

Table 3

\section{CONCLUSION}

In this paper non-linear companding transform has been proposed and evaluated for OFDM employing 16 QAM and 64 QAM signal mapping. The effect of applying the proposed NCT has been observed for different constellation sizes. In both the conditions the proposed NCT reduces the PAPR significantly for the OFDM signal which shows that NCT provides a flexible method for reduction of PAPR in OFDM system. The proposed NCT has the capability to effectively reduce the inherent problem of high PAPR in OFDM systems. The companding function is effective as well as flexible as it is able to reduce PAPR for different constellation sizes. The proposed NCT is able to transform the amplitude or power of the original OFDM signal into uniform distribution. The original OFDM signal is Gaussian distributed. By the application of NCT Gaussian distribution is converted into a specific statistics as defined by the PDF of the NCT. The proposed NCT can be further evaluated on parameters like BER by applying the appropriate channel models.

\section{REFERENCES}

[1]. M Hongwei Yang, "A road to future broadband wireless access: MIMO-OFDM based air interface," IEEE Communications Magazine, Vol.43, No.1, pp. 53-60, Feb 2005.

Y.Wu, W. Y. Zou, "Orthogonal frequency division multiplexing: a multi-carrier modulation scheme," IEEE Transactions on Consumer Electronics, Vol. 41, No. 3, pp. 392-399, Aug. 1995.

S. H. Han, J. H. Lee, "An overview of peak-to-average power ratio reduction techniques for multicarrier transmission," IEEE Wireless Communications Magazine, Vol 12, No.2, pp. 55-65, April 2005.

[4] B.Wang, T. T. Tjhung, and C. S. Ng, "Reduction of peak-to-average power ratio of OFDM system using a companding technique," IEEE Transactions on Broadcasting, Vol. 45, No. 3, pp. 303-307, Sep. 1999.

[5] J. Hou, J. Ge, D. Zhai, and J. Li, "Peak-to-average power ratio reduction of OFDM signals with nonlinear companding scheme", IEEE Tranasctions on Broadcasting, Vol. 56, No. 2, pp. 258-262, June 2010.

[6] T. Jiang and Y.Wu, "An overview: Peak-to-average power ratio reduction techniques for OFDM signals," IEEE Transactions on Broadcasting, Vol. 54, No. 2, pp. 257-268, Jun. 2008.

D. Dardari, V. Tralli, and A. Vaccari, "A theoretical characterization of nonlinear distortion effects in OFDM systems," IEEE Transactions on Communication, Vol. 48, No. 4, pp. 1755-1764, Oct. 2000.

P. Banelli and S. Cacopardi, "Theoretical analysis and performance of OFDM signals in nonlinear AWGN channels," IEEE Transactions on Communication, Vol. 48, No.2, pp. 430-441, Mar. 2000.

A. Mattsson, G. Mendenhall, and T. Dittmer, "Reduction of peak-to-average power ratio of ofdm system using a companding technique," IEEE Transactions on Broadcasting, Vol. 45, No.6, pp. 418-419, Dec. 1999.

[10]. S. A. Aburakhiya, E. F. Badran, D. A. E Mohamed, "Non-linear companding transform for reduction of peak-to-average power ratio of OFDM signal," IEEE Transactions on Broadcasting, Vol 55, No.1, pp 456-461, March 2009.

[11]. Y. Rahamatallah, N. Bouaynaya, S. Mohan, "On the performance of linear and non-linear companding transforms in OFDM systems," IEEE Wireless Telecommunication Symposium, pp.1-5, April 2011.

[12]. X. Huang, J. Lu, J.Zheng, K. B. Letaief, J. Gu, " Companding transform for reduction in peak-to-average power ratio of OFDM signals", IEEE Transactions on Wireless Communications, Vol. 03, No. 6, pp.2030-2039, Nov 2004.

[13]. Y.Z.Jiao, X.j. Liu and X.A. Wang, " a novel Tone reservation scheme with fast covergance for PAPR reduction in OFDM systems," IEEE communication society, pp. 398-402, Aug 2008.

[14]. S. -S. Jeng, J. -M. Chen, "Implementation of low complexity companding technique for effiecient peak to average ratio reduction in orthogonal frequency division multiplexing systems", IEEE Transactions on Broadcating, Vol. 5, No. 2, pp. 154-162, 2011.

[15]. S.-S.Jeng, J.-M. Chen, "Efficient PAPR reduction in OFDM systems based on a companding technique with trapezium distribution", IEEE Transactions on Broadcasting, Vol. 5, No. 2, pp. 291-298, June 2011.

[16]. N.S.L Phani Kumar, A. Banerjee, Pardeep Sircar, "Modified exponential companding for PAPR Reduction of OFDM signals," IEEE Communication Society proceedings, pp. 1345-1350, August 2007.

[17]. Vipin Uppal and Garima Saini, "Reduction of peak-to-average-power-ratio in OFDM signal using Non-Linear Transform," International Journal of Electrical,Electronics and Communication Engg., Vol.1, No. 3, pp 123-129, May 2011.

[18] Hsinying Liang and Yan Ru Chen "A modified genetic algorithm PTS technique for PAPR reduction in OFDM systems",Asia Pacific Conference on Communication, pp 182-185, July 2009. 